\title{
Maps of Attractiveness of European Regions
}

\author{
Otakar Čerba* \\ Department of Geomatics, University of West Bohemia, Plzen̆, Czech Republic, Otakar Čerba - cerba@kgm.zcu.cz. \\ * Corresponding author
}

Keywords: map, cartography, attractiveness, rural region

\begin{abstract}
:
European society is facing many critical challenges. Traditional as well as social media, inform people about a particular crisis. The news titles during the last years have contained words such as COVID-19, drought, floods or migration. The society, including experts, call for a quick and straightforward solution of particular burning troubles. However, all of the problems afflicting contemporary Europe is much more complex. The regional development is a keystone of problems mentioned-above. Visions of regional development, as well as efficient cooperation and sharing knowledge, can prevent or limit the impact of various types of hazards and risks. Prosperous and vital regions can help with many burning problems threatening the human population such as climate change, poverty, hunger, health or clean energy. The information is a crucial commodity from the perspective of regional development. Such information has to be high-quality and reliable. The way of presentation and interpretation is also critical. Therefore the maps of the regional attractiveness (which is taken as a potential region) have been developed in the Polirural project. The article "Maps of Attractiveness of European Regions" presents two types of thematic maps of regional attractiveness: choropleth maps providing information on a level of attractiveness based on Rural Attractiveness Index (RAI) and chorochromatic maps showing similarities on regions calculated by clustering techniques. These maps cover NUTS 3 region in Europe. The target group for the developed map is very heterogeneous. It can cover policy-makers and decision-makers of particular regions; people focused on regional development and planning or investments, persons interested in entrepreneurship or citizen living in particular areas. Because of the variety of user group and limited experience with advanced cartographic products, the developed maps have to be simple, clear, comparable, intelligible and attractive.
\end{abstract}

